Document downloaded from:

http://hdl.handle.net/10251/43720

This paper must be cited as:

Gutierrez García, J.; Romaguera Bonilla, S.; Sanchis, M. (2013). An identification theorem for the completion of the Hausdorff fuzzy metric. Fuzzy Sets and Systems. 227:96-106. doi:10.1016/j.fss.2013.04.012.

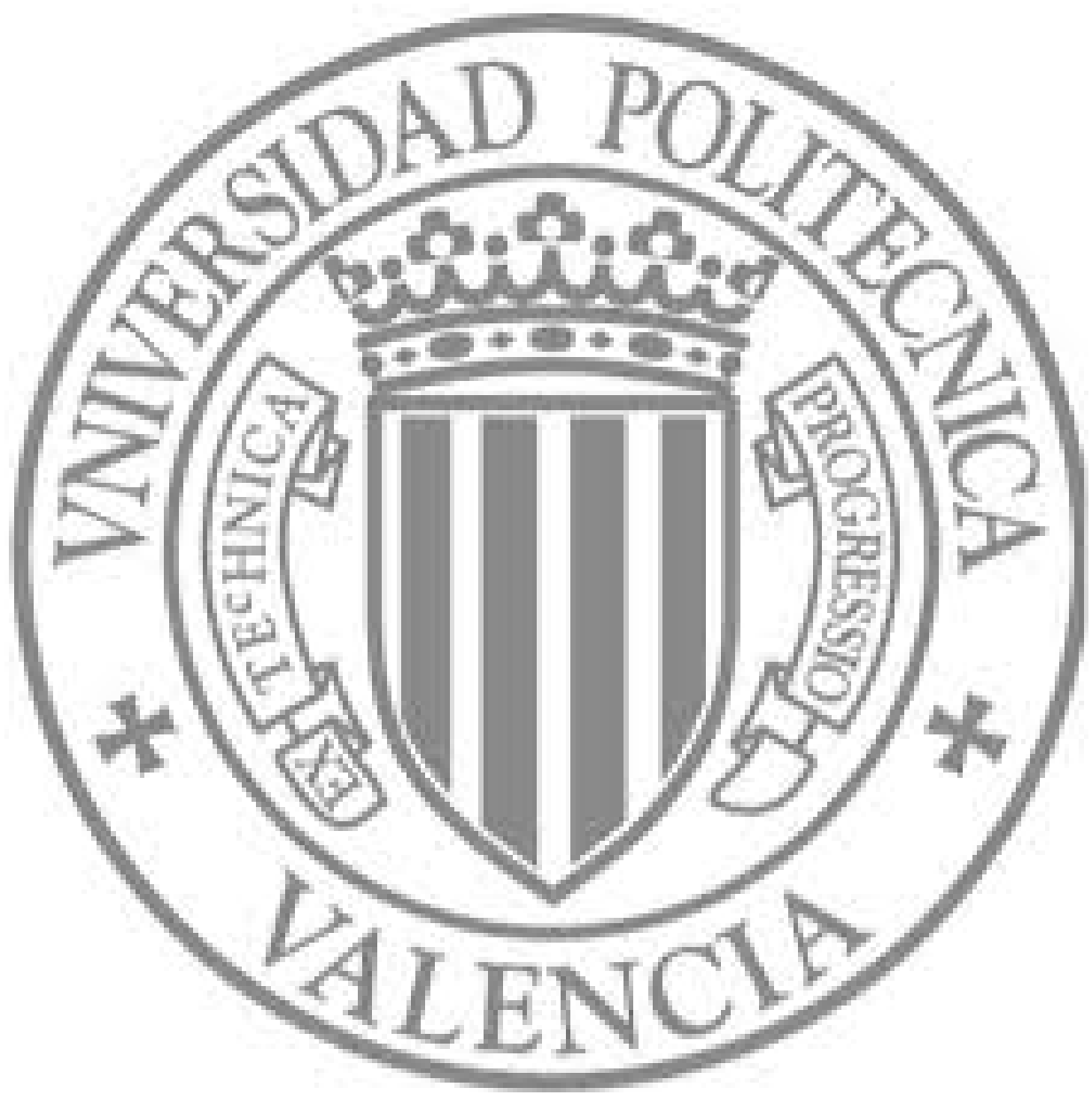

The final publication is available at

http://dx.doi.org/10.1016/j.fss.2013.04.012

Copyright Elsevier 


\title{
An identification theorem for the completion of the Hausdorff fuzzy metric
}

\author{
J. Gutiérrez García ${ }^{a}$, S. Romaguera ${ }^{b, *}$, M. Sanchis ${ }^{c}$ \\ ${ }^{a}$ Departamento de Matemáticas, Universidad del País Vasco UPV/EHU, \\ Apdo. 644, 48080, Bilbao, Spain \\ ${ }^{b}$ Instituto Universitario de Matemática Pura y Aplicada, Universitat Politècnica de València, \\ Camí de Vera s/n, 46022 Valencia, Spain \\ ${ }^{c}$ Institut Universitari de Matemàtiques i Aplicacions de Castelló (IMAC) Universitat Jaume I, \\ Campus del Riu Sec. s/n, 12071 Castelló, Spain
}

\begin{abstract}
We prove that given a fuzzy metric space (in the sense of Kramosil and Michalek), the completion of its Hausdorff fuzzy metric space is isometric to the Hausdorff fuzzy metric space of its completion, when the Hausdorff fuzzy metrics are defined on the respective collections of non-empty closed subsets. As a consequence, we deduce the corresponding result for metric spaces by using the standard fuzzy metric. An application to the extension of multivalued mappings and some illustrative examples are also given.
\end{abstract}

Keywords: Hausdorff fuzzy metric, Completion, Isometry, Contraction, Dynamical System.

\section{Introduction and preliminaries}

Throughout this paper the letters $\mathbb{N}$ and $\omega$ will denote the set of positive integer numbers and the set of non-negative integer numbers, respectively. By $C_{0}(X)$ and $\mathcal{K}_{0}(X)$ we will denote the collection of non-empty closed subsets and the collection of non-empty compact subsets of a topological space $X$, respectively. If

\footnotetext{
${ }^{2}$ Research supported by the Ministry of Economy and Competitiveness of Spain, under grants MTM2012-37894-C02-01 and MTM2012-37894-C02-02. The first named author also acknowledges financial support from the UPV/EHU under grants UFI 11/52 and GIU12/39.

* Corresponding author

Email addresses: javier.gutierrezgarcia@ehu.es (J. Gutiérrez García), sromague@mat.upv.es (S. Romaguera), sanchis@mat.uji.es (M. Sanchis)
} 
$A$ is a subset of a topological space $X$, we will denote by $\operatorname{cl}_{X} A$, or by $\bar{A}$, the closure of $A$ in $X$.

In order to help to the reader, we first recall several pertinent concepts and results on the Hausdorff distance of a metric space and on the Hausdorff fuzzy metric of a fuzzy metric space, respectively.

By an infinite valued metric on a set $X$ we mean a function $d: X \times X \rightarrow[0,+\infty]$ that satisfies the usual axioms of a metric, except that we allow $d(x, y)=+\infty$.

Thus, in our context, a metric space will be a pair $(X, d)$ such that $X$ is a (nonempty) set and $d$ is a (possibly, infinite valued) metric on $X$.

The completion of a metric space $(X, d)$, as constructed for instance in [17, Theorem 1.15$]$, will be denoted by $(\widetilde{X}, \widetilde{d})$.

Let us recall that, given a metric space $(X, d)$, the Hausdorff distance on $C_{0}(X)$ is defined as the infinite valued metric $H_{d}: C_{0}(X) \times C_{0}(X) \rightarrow[0,+\infty]$ given by $H_{d}=\max \left\{H_{d}^{-}, H_{d}^{+}\right\}$, where $H_{d}^{-}(A, B)=\sup _{a \in A} d(a, B)$ and $H_{d}^{+}(A, B)=\sup _{b \in B} d(A, b)$, for all $A, B \in C_{0}(X)$ (see, for instance, [4, Section 3.2]).

In [20, Proposition 4.1] Michael proved that a metric space $(X, d)$ is complete if and only if $\left(C_{0}(X), H_{d}\right)$ is complete. Later on, Artico and Moresco showed in [2, Proposition 1.3] that for any metric space $(X, d)$, the complete metric spaces $\left(\widetilde{C_{0}(X)}, \widetilde{H_{d}}\right)$ and $\left(C_{0}(\widetilde{X}), H_{\widetilde{d}}\right)$ are uniformly isomorphic.

According to [28], a continuous t-norm is a binary operation $*:[0,1] \times[0,1] \rightarrow$ $[0,1]$ which satisfies the following conditions: (i) $*$ is associative and commutative; (ii) $*$ is continuous; (iii) $a * 1=a$ for every $a \in[0,1]$; (iv) $a * b \leq c * d$ whenever $a \leq c$ and $b \leq d$, and $a, b, c, d \in[0,1]$.

Three paradigmatic examples of continuous t-norms are $\wedge$, Prod and $*_{L}$ (the Lukasiewicz t-norm), which are defined by $a \wedge b=\min \{a, b\}, a \operatorname{Prod} b=a b$, and $a *_{L} b=\max \{a+b-1,0\}$ for all $a, b \in[0,1]$, respectively. Recall that $* \leq \wedge$ for every continuous t-norm $*$, and that continuous t-norm greater or equal than $*_{L}$ is a copula [18].

By a fuzzy metric on a set $X$ (compare [19, Definition 7]) we mean a pair $(M, *)$ such that $*$ is a continuous t-norm and $M$ is a fuzzy set in $X \times X \times[0,+\infty)$ such that for all $x, y, z \in X$ :

(i) $M(x, y, 0)=0$;

(ii) $x=y$ if and only if $M(x, y, t)=1$ for all $t>0$;

(iii) $M(x, y, t)=M(y, x, t)$;

(iv) $M(x, z, t+s) \geq M(x, y, t) * M(y, z, s)$ for all $t, s \geq 0$;

(v) $M(x, y, \cdot):[0,+\infty) \rightarrow[0,1]$ is left continuous. 
A fuzzy metric space is a triple $(X, M, *)$ such that $X$ is a set and $(M, *)$ is fuzzy metric on $X$.

It is well known, and easy to see, that for all $x, y \in X, M(x, y, \cdot)$ is a nondecreasing function on $[0,+\infty)$.

Each fuzzy metric $(M, *)$ on a set $X$ induces a topology $\tau_{M}$ on $X$ which has as a base the family of open balls $\left\{B_{M}(x, \varepsilon, t): x \in X, \varepsilon \in(0,1), t>0\right\}$, where $B_{M}(x, \varepsilon, t)=\{y \in X: M(x, y, t)>1-\varepsilon\}$.

It is also well known (see, for instance, [13]) that every fuzzy metric space $(X, M, *)$ is metrizable, i.e., there exists a metric $d$ on $X$ whose induced topology agrees with $\tau_{M}$.

Conversely, if $(X, d)$ is a metric space and we define $M_{d}: X \times X \times[0,+\infty) \rightarrow$ $[0,1]$ by $M_{d}(x, y, 0)=0$ and

$$
M_{d}(x, y, t)=\frac{t}{t+d(x, y)},
$$

for all $t>0$, then $\left(M_{d}, *\right)$ is a fuzzy metric on $X$ called the standard fuzzy metric of $(X, d)$ (compare [11]). Moreover, the topology $\tau_{M_{d}}$ agrees with the topology induced by $d$.

A fuzzy metric space $(X, M, *)$ is said to be complete [12] if every Cauchy sequence converges with respect to $\tau_{M}$, where a sequence $\left(x_{n}\right)_{n \in \mathbb{N}}$ is Cauchy provided that for each $t>0$ and each $\varepsilon \in(0,1)$ there exists $n_{0} \in \mathbb{N}$ such that $M\left(x_{n}, x_{m}, t\right)>1-\varepsilon$ for all $n, m \geq n_{0}$. If $(X, M, *)$ is complete we say that the fuzzy metric $(M, *)$ is complete.

A mapping $f$ from a fuzzy metric space $(X, M, *)$ to a fuzzy metric space $(Y, N, \diamond)$ is said to be an isometry ([14]) if $M(x, y, t)=N(f(x), f(y), t)$ for all $x, y \in X, t>0$. Two fuzzy metric spaces $(X, M, *)$ and $(Y, N, \diamond)$ are isometric provided that there exists an isometry from $(X, M, *)$ onto $(Y, N, \diamond)$.

Similarly to the classical metric case, by a completion of a fuzzy metric space $(X, M, *)$ we mean a complete fuzzy metric space $(Y, N, \diamond)$ that has a dense subspace isometric to $(X, M, *)$.

It is well known (see, for instance, [7, Remark 3]) that each fuzzy metric space has a completion which is unique up to isometry. This is a consequence of the fact, proved by Sherwood ([30]), that every Menger space, with continuous t-norm, has a completion which is unique up to isometry, and of the "equivalence" between fuzzy metric spaces and Menger spaces with continuous t-norm, observed by Kramosil and Michalek in [19, Theorem 1].

In the sequel, we will denote by $(\widetilde{X}, \widetilde{M}, *)$ the completion of the fuzzy metric space $(X, M, *)$ as constructed in [7, Theorem 1 and Remark 3], and we will refer 
to $(\widetilde{M}, *)$ as the completion of the fuzzy metric $(M, *)$.

We briefly recall such a construction. Given the fuzzy metric space $(X, M, *)$, denote by $S$ the collection of all Cauchy sequences in $(X, M, *)$. For each $\left(x_{n}\right)_{n \in \mathbb{N}} \in$ $S$, put

$$
\left[\left(x_{n}\right)_{n \in \mathbb{N}}\right]=\left\{\left(y_{n}\right)_{n \in \mathbb{N}} \in S: \lim _{n \rightarrow \infty} M\left(x_{n}, y_{n}, t\right)=1 \text { for all } t>0\right\},
$$

and let $\widetilde{X}=\left\{\left[\left(x_{n}\right)_{n \in \mathbb{N}}\right]:\left(x_{n}\right)_{n \in \mathbb{N}} \in S\right\}$.

Then [7, Theorem 1 and Remark 2.11], the pair $(\widetilde{M}, *)$ is a complete fuzzy metric on $\widetilde{X}$, where $\widetilde{M}: \widetilde{X} \times \widetilde{X} \times[0,+\infty) \rightarrow[0,1]$ is defined for all $\left(x_{n}\right)_{n \in \mathbb{N}},\left(y_{n}\right)_{n \in \mathbb{N}} \in$ $S$ by $\widetilde{M}\left(\left[\left(x_{n}\right)_{n \in \mathbb{N}}\right],\left[\left(y_{n}\right)_{n \in \mathbb{N}}\right], t\right)=0$ and

$$
\widetilde{M}\left(\left[\left(x_{n}\right)_{n \in \mathbb{N}}\right],\left[\left(y_{n}\right)_{n \in \mathbb{N}}\right], t\right)=\sup _{0<s<t} \sup _{k \in \mathbb{N}} \inf _{n \geq k} M\left(x_{n}, y_{n}, s\right)
$$

for every $t>0$. Moreover $(\widetilde{X}, \widetilde{M}, *)$ has a dense subspace which is isometric to $(X, M, *)$, and every completion of $(X, M, *)$ is isometric to $(\widetilde{X}, \widetilde{M}, *)$.

Next we proceed to recall the construction of the Hausdorff fuzzy metric of a given fuzzy metric space $(X, M, *)$ (see e.g. [24, p. 1082]). In fact, it can be obtained as a simple adaptation to the fuzzy setting of the definition of the Hausdorff probabilistic metric of a probabilistic metric space $([9,28,29,31])$.

Given $x \in X, A \subseteq X$ non-empty and $t>0$, set $M(x, A, t)=\sup _{a \in A} M(x, a, t)$. For each $A, B \in C_{0}(X)$ let

$$
\begin{aligned}
& H_{M}^{-}(A, B, 0)=H_{M}^{+}(A, B, 0)=0, \\
& H_{M}^{-}(A, B, t)=\sup _{0<s<t} \inf _{a \in A} M(a, B, s), H_{M}^{+}(A, B, t)=\sup _{0<s<t} \inf _{b \in B} M(A, b, s) \quad \text { for every } t>0, \\
& \text { and } \\
& H_{M}(A, B, t)=\min \left\{H_{M}^{-}(A, B, t), H_{M}^{+}(A, B, t)\right\} \quad \text { for every } t \geq 0 .
\end{aligned}
$$

Then $\left(H_{M}, *\right)$ is a fuzzy metric on $C_{0}(X)$, called the Hausdorff fuzzy metric of $(X, M, *)$.

The fuzzy metric space $\left(C_{0}(X), H_{M}, *\right)$ is said to be the Hausdorff fuzzy metric space of $(X, M, *)$ or the fuzzy hyperspace of $(X, M, *)$.

Similarly to the classical metric case, one has that a fuzzy metric space $(X, M, *)$ is complete if and only if $\left(C_{0}(X), H_{M}, *\right)$ is complete (see e.g. [24, Corolllary of Theorem 5]).

Consequently, if, on the one hand, we construct on $C_{0}(\widetilde{X})$ the (complete) Hausdorff fuzzy metric $\left(H_{\widetilde{M}}, *\right)$ of $(\widetilde{X}, \widetilde{M}, *)$ and, on the other hand, we construct on $\widetilde{C_{0}(X)}$ the completion $\left(\widetilde{H_{M}}, *\right)$ of the Hausdorff fuzzy metric $\left(H_{M}, *\right)$, the following 
question arises in a natural way: Find the relationship between the fuzzy metric spaces $\left(C_{0}(\widetilde{X}), H_{\widetilde{M}}, *\right)$ and $\left(\widetilde{C_{0}(X)}, \widetilde{H_{M}}, *\right)$.

Answering this question we shall prove that such spaces are isometric. From this result, and with the help of the standard fuzzy metric, we shall deduce that given a metric space $(X, d)$, then the (complete) metric spaces $\left(\widetilde{C_{0}(X)}, \widetilde{H_{d}}\right)$ and $\left(C_{0}(\widetilde{X}), H_{\widetilde{d}}\right)$ are isometric, improving in this way Artico-Moresco's result mentioned above. An application to the extension of multivalued mappings, via a version of Nadler's fixed point theorem [21], and some illustrative examples are also given.

\section{The main results}

In order to obtain our main results, the following auxiliary technical lemmas will be useful.

2.1 Lemma. Let $(X, M, *)$ be a fuzzy metric space. Then, for each $a \in X$ and each non-empty subset $B$ of $X$, the function $M(a, B, \cdot):[0,+\infty) \rightarrow[0,1]$ is left continuous.

Proof. It follows immediately from the facts that $M(a, B, \cdot)$ is non-decreasing and $M(a, b, \cdot)$ is left continuous for each $b \in B$.

2.2 Lemma. Let $(X, M, *)$ be a fuzzy metric space. Then, for each $a \in X$ and each non-empty subset $B$ of $X$, it follows that $M(a, B, t)=M(a, \bar{B}, t)$.

Proof. Since $B \subseteq \bar{B}$ we obviously obtain that $M(a, B, t) \leq M(a, \bar{B}, t)$.

In order to show that $M(a, B, t) \geq M(a, \bar{B}, t)$ we shall assume that $M(a, \bar{B}, t)>$ 0 . By the preceding lemma there exists $\varepsilon_{0} \in(0, t)$ such that $M(a, \bar{B}, t-\varepsilon)>0$ whenever $\varepsilon \in\left(0, \varepsilon_{0}\right)$. Choose an arbitrary $\varepsilon \in\left(0, \varepsilon_{0}\right)$. Then, for each $\delta \in(0, \varepsilon)$ with $M(a, \bar{B}, t-\varepsilon)>\delta$ there exists $x \in \bar{B}$ such that

$$
M(a, \bar{B}, t-\varepsilon)<M(a, x, t-\varepsilon)+\delta .
$$

Let $b \in B$ such that $M(x, b, \varepsilon)>1-\delta$. Therefore

$M(a, B, t) \geq M(a, b, t) \geq M(a, x, t-\varepsilon) * M(x, b, \varepsilon) \geq(M(a, \bar{B}, t-\varepsilon)-\delta) *(1-\delta)$.

By continuity of $*$ we deduce that $M(a, B, t) \geq M(a, \bar{B}, t-\varepsilon)$ for all $\varepsilon \in\left(0, \varepsilon_{0}\right)$. So, by Lemma $2.1, M(a, B, t) \geq M(a, \bar{B}, t)$, which completes the proof.

The following is a direct consequence of Lemma 2.2. 
2.3 Lemma. Let $(X, M, *)$ be a fuzzy metric space. Then, for each pair $A, B$ of non-empty subsets of $X$ and each $t>0$ it follows that

$$
\inf _{x \in \bar{A}} M(x, \bar{B}, t) \leq \inf _{a \in A} M(a, B, t) .
$$

2.4 Lemma. Let $(X, M, *)$ be a fuzzy metric space and let $\phi: C_{0}(X) \rightarrow C_{0}(\widetilde{X})$ be given by $\phi(A)=\operatorname{cl}_{\widetilde{X}} A$ for all $A \in \mathcal{C}_{0}(X)$. Then the fuzzy metric spaces $\left(C_{0}(X), H_{M}, *\right)$ and $\left(\phi\left(C_{0}(X)\right), H_{\widetilde{M}}, *\right)$ are isometric.

Proof. Let $A, B \in C_{0}(X)$ and $t>0$.

We first prove that $H_{M}^{-}(A, B, t)=H_{\widetilde{M}}^{-}(\phi(A), \phi(B), t)$. Indeed, given $s \in(0, t)$, it follows from Lemma 2.3, that

$$
\inf _{x \in \mathrm{cl}_{\widetilde{X}} A} \widetilde{M}\left(x, \mathrm{cl}_{\widetilde{X}} B, s\right) \leq \inf _{a \in A} \widetilde{M}(a, B, s) .
$$

So

$$
H_{\widetilde{M}}^{-}(\phi(A), \phi(B), t)=\sup _{0<s<t} \inf _{x \in \mathrm{cl}_{\widetilde{X}} A} \widetilde{M}\left(x, \mathrm{cl}_{\widetilde{X}} B, s\right) \leq \sup _{0<s<t} \inf _{a \in A} \widetilde{M}(a, B, s)=H_{M}^{-}(A, B, t) .
$$

Next we show that $H_{M}^{-}(A, B, t) \leq H_{\widetilde{M}}^{-}(\phi(A), \phi(B), t)$.

Let $s \in(0, t)$. For each $\varepsilon \in(0, \min \{t-s, 1\})$ there is $x_{\varepsilon} \in \phi(A)$ such that

$$
\inf _{x \in \phi(A)} \widetilde{M}(x, \phi(B), s+\varepsilon)+\varepsilon \geq \widetilde{M}\left(x_{\varepsilon}, \phi(B), s+\varepsilon\right) .
$$

Since $x_{\varepsilon} \in \phi(A)$ there is $a_{\varepsilon} \in A$ such that $\widetilde{M}\left(x_{\varepsilon}, a_{\varepsilon}, \varepsilon\right)>1-\varepsilon$. Hence $\widetilde{M}\left(x_{\varepsilon}, \phi(B), s+\varepsilon\right) \geq \widetilde{M}\left(x_{\varepsilon}, B, s+\varepsilon\right) \geq \widetilde{M}\left(x_{\varepsilon}, a_{\varepsilon}, \varepsilon\right) * \widetilde{M}\left(a_{\varepsilon}, B, s\right) \geq(1-\varepsilon) * M\left(a_{\varepsilon}, B, s\right)$.

Taking limits when $\varepsilon \rightarrow 0$, we deduce by the continuity of $*$, that

$$
\sup _{0<r<t} \inf _{x \in \phi(A)} \widetilde{M}(x, \phi(B), r) \geq \inf _{a \in A} M(a, B, s)
$$

whenever $s \in(0, t)$. Thus

$$
H_{\widetilde{M}}^{-}(\phi(A), \phi(B), t) \geq H_{M}^{-}(A, B, t) .
$$

Finally, since $H_{M}^{+}(A, B, t)=H_{M}^{-}(B, A, t)$, we deduce from the equality proved above that $H_{M}^{+}(A, B, t)=H_{\widetilde{M}}^{+}(\phi(A), \phi(B), t)$.

Therefore $\phi$ is an isometry from $\left(C_{0}(X), H_{M}, *\right)$ onto $\left(\phi\left(C_{0}(X)\right), H_{\widetilde{M}}, *\right)$. This concludes the proof. 
2.5 Theorem. Let $(X, M, *)$ be a fuzzy metric space. Then the (complete) fuzzy metric spaces $\left(C_{0}(\widetilde{X}), H_{\widetilde{M}}, *\right)$ and $\left(\widetilde{C_{0}(X)}, \widetilde{H_{M}}, *\right)$ are isometric.

Proof. Let $\phi: \mathcal{C}_{0}(X) \rightarrow C_{0}(\widetilde{X})$ as defined in Lemma 2.4. We show that $\phi\left(C_{0}(X)\right)$ is dense in $\left(C_{0}(\widetilde{X}), H_{\widetilde{M}}, *\right)$. Indeed, let $B \in C_{0}(\widetilde{X}), \varepsilon \in(0,1)$ and $t>0$. Since $X$ is dense in $(\widetilde{X}, \widetilde{M}, *)$, for each $b \in B$ there is $x_{b} \in X$ such that $\widetilde{M}\left(b, x_{b}, t / 2\right)>1-\varepsilon$. Put $A=\operatorname{cl}_{X}\left(\left\{x_{b}: b \in B\right\}\right)$. Then, it is routine to check that $\phi(A) \in H_{\widetilde{M}}(B, \varepsilon, t)$. Hence $\phi\left(C_{0}(X)\right)$ is dense in the complete fuzzy metric space $\left(C_{0}(\widetilde{X}), H_{\widetilde{M}}, *\right)$.

Finally, it follows from Lemma 2.4 that $\left(C_{0}(\widetilde{X}), H_{\widetilde{M}}, *\right)$ is (isometric to the) completion of $\left(C_{0}(X), H_{M}, *\right)$.

2.6 Remark. Notice that the restriction of the mapping $\phi$, constructed in Lemma 2.4, to $\mathcal{K}_{0}(X)$ is the identity. Now, since $X$ is dense in $(\widetilde{X}, \vec{M}, *)$, given $\varepsilon \in(0,1), t>0$ and a finite subset $B=\left\{\widetilde{x}_{1}, \widetilde{x}_{2}, \ldots, \widetilde{x}_{n}\right\}$ of $\widetilde{X}$, there exists $A=\left\{x_{1}, x_{2}, \ldots, x_{n}\right\} \subset$ $X$ such that, for every $i=1,2, \ldots, n$, we have $\widetilde{M}\left(\widetilde{x}_{i}, x_{i}, t / 2\right)>1-\varepsilon$. Thus, $\phi(A) \in H_{\widetilde{M}}(B, \varepsilon, t)$. Taking into account that the set of all finite subsets of $\mathcal{K}_{0}(\widetilde{X})$ is dense, we have just showed that $\mathcal{K}_{0}(X)$ is dense in the complete fuzzy metric space $\left(\mathcal{K}_{0}(\widetilde{X}), H_{\widetilde{M}}, *\right)$. Thus, Theorem 2.5 remains valid if $C_{0}(X)$ is replaced by $\mathcal{K}_{0}(X)$ and $C_{0}(\widetilde{X})$ by $\mathcal{K}_{0}(\widetilde{X})$.

In [21], Nadler proved his celebrated fixed point theorem that if $(X, d)$ is a complete metric space and $T: X \rightarrow \mathcal{C}_{0}(X)$ is a multivalued mapping with the property that there is $\alpha \in(0,1)$ such that

$$
H_{d}(T x, T y) \leq \alpha d(x, y)
$$

for all $x, y \in X$, then $T$ has a fixed point, i.e., there is $z \in X$ satisfying $z \in T z$.

Our next goal is to give a suitable version of Nadler's fixed point theorem to our framework and then apply it, together with Theorem 2.5 above, to study the extension of a multivalued mapping on a fuzzy metric space to its completion.

There exist several fuzzy generalizations of Nadler's fixed point theorem in the literature; in fact, excellent contributions to this question may be found in $[22,15]$. However, the result stated in Proposition 2.7 below will be sufficient for our purposes.

According to Hicks [16], a self mapping $f$ of a fuzzy metric space $(X, M, *)$ is called a $C$-contraction if there is $\alpha \in(0,1)$ such that for all $x, y \in X$ and each $t>0$, the following implication holds:

$$
M(x, y, t)>1-t \Longrightarrow M(f x, f y, \alpha t)>1-\alpha t .
$$


Pap, Hadžić and Mesiar [22] generalized $C$-contractions to multivalued mappings introducing the notion of a $(\Psi, C)$-contraction. We adapt this notion, to our context, as follows.

Let $(X, M, *)$ be a fuzzy metric space and let $T: X \rightarrow C_{0}(X)$ be a multivalued mapping. Given $\Psi:[0,+\infty) \rightarrow[0,+\infty)$, we say that $T$ is a $(\Psi, C)$-contraction on $(X, M, *)$ if for each $x, y \in X$ and each $t>0$, the following implication holds:

$$
M(x, y, t)>1-t \Longrightarrow H_{M}(T x, T y, \Psi(t))>1-\Psi(t) .
$$

By an $(\alpha, C)$-contraction we mean a $(\Psi, C)$-contraction such that $\Psi(t)=\alpha t$ for all $t \in \mathbb{R}^{+}$, where $\alpha$ is a constant with $0<\alpha<1$. Then, we have the following result.

2.7 Proposition. Let $(X, M, *)$ be a complete fuzzy metric space with $*_{L} \leq *$. Then, every $(\alpha, C)$-contraction on $(X, M, *)$ has a fixed point.

Proof. Let $T: X \rightarrow C_{0}(X)$ be an $(\alpha, C)$-contraction on $(X, M, *)$. Fix $x_{0} \in X$ and $t_{0}>1$. Take $x_{1} \in T x_{0}$. Then $M\left(x_{0}, x_{1}, t_{0}\right)>1-t_{0}$, so, there exists $x_{2} \in T x_{1}$ such that $M\left(x_{1}, x_{2}, \alpha t_{0}\right)>1-\alpha t_{0}$. Similarly, there exists $x_{3} \in T x_{2}$ such that $M\left(x_{2}, x_{3}, \alpha^{2} t_{0}\right)>1-\alpha^{2} t_{0}$. Repeating this process, we obtain a sequence $\left(x_{n}\right)_{n \in \omega}$ with $x_{n+1} \in T x_{n}$ and

$$
M\left(x_{n}, x_{n+1}, \alpha^{n} t_{0}\right)>1-\alpha^{n} t_{0},
$$

for all $n \in \omega$. We show that $\left(x_{n}\right)_{n \in \omega}$ is a Cauchy sequence in $(X, M, *)$. Indeed, given $t>0$ and $\varepsilon \in(0, t)$ there exists $n_{0} \in \mathbb{N}$ such that $\sum_{m=n_{0}}^{\infty} \alpha^{m} t_{0}<\varepsilon$. Then, for each $n \geq n_{0}$ and each $j \in \mathbb{N}$ we obtain

$$
\begin{aligned}
M\left(x_{n}, x_{n+j}, t\right) & \geq M\left(x_{n}, x_{n+j}, \varepsilon\right) \geq M\left(x_{n}, x_{n+j}, \sum_{m=n}^{n+j-1} \alpha^{m} t_{0}\right) \\
& \geq M\left(x_{n}, x_{n+1}, \alpha^{n} t_{0}\right) * \cdots * M\left(x_{n+j-1}, x_{n+j}, \alpha^{n+j-1} t_{0}\right) \\
& \geq M\left(x_{n}, x_{n+1}, \alpha^{n} t_{0}\right) *_{L} \cdots *_{L} M\left(x_{n+j-1}, x_{n+j}, \alpha^{n+j-1} t_{0}\right) \\
& \geq\left(1-\alpha^{n} t_{0}\right) *_{L} \cdots *_{L}\left(1-\alpha^{n+j-1} t_{0}\right) \\
& =1-\sum_{m=n}^{n+j-1} \alpha^{m} t_{0}>1-\varepsilon .
\end{aligned}
$$

So $\left(x_{n}\right)_{n \in \omega}$ is a Cauchy sequence in $(X, M, *)$. Hence, there is $z \in X$ such that $\left(x_{n}\right)_{n \in \omega}$ converges to $z$ with respect to $\tau_{M}$, i.e., $\lim _{n} M\left(z, x_{n}, t\right)=1$ for every $t>0$.

We show that $z \in T z$. Indeed, for each $n \in \mathbb{N}$ there exists $j_{n}>n$ such that $M\left(z, x_{m}, \frac{1}{n}\right)>1-\frac{1}{n}$ for every $m \geq j_{n}$, so for each $n \in \mathbb{N}$ there exists $z_{n} \in T z$ such that $M\left(z_{n}, x_{j_{n}+1}, \frac{\alpha}{n}\right)>1-\alpha / n$. Since for each $n \in \mathbb{N}$

$$
M\left(z, z_{n}, \frac{\alpha+1}{n}\right) \geq M\left(z, x_{j_{n}+1}, \frac{1}{n}\right) * M\left(x_{j_{n}+1}, z_{n}, \frac{\alpha}{n}\right) \geq\left(1-\frac{1}{n}\right) *\left(1-\frac{\alpha}{n}\right),
$$


we deduce that $\left(z_{n}\right)_{n \in \mathbb{N}}$ converges to $z$ with respect to $\tau_{M}$. Therefore $z \in \overline{T z}=T z$, i.e., $z$ is a fixed point of $T$.

2.8 Remark. Note that Proposition 2.7 remains true for any $(\Psi, C)$-contraction such that the function $\Psi$ is non-decreasing and satisfies $\sum_{n=0}^{\infty} \Psi^{n}(t)<\infty$ for all $t>0$ (compare [22, Theorem 1]).

Now suppose that $(X, M, *)$ is a fuzzy metric space and $T: X \rightarrow C_{0}(X)$ an $(\alpha, C)$-contraction. In a natural way, we extend $T$ to the completion of $(X, M, *)$ as follows.

For each Cauchy sequence $\left(x_{n}\right)_{n \in \mathbb{N}}$ in $(X, M, *)$ put

$$
\widetilde{T}\left[\left(x_{n}\right)_{n \in \mathbb{N}}\right]=\left[\left(T x_{n}\right)_{n \in \mathbb{N}}\right] .
$$

Note that if $\left(y_{n}\right)_{n \in \mathbb{N}} \in\left[\left(x_{n}\right)_{n \in \mathbb{N}}\right]$, then $\widetilde{T}\left[\left(y_{n}\right)_{n \in \mathbb{N}}\right]=\widetilde{T}\left[\left(x_{n}\right)_{n \in \mathbb{N}}\right]$.

Moreover $\widetilde{T}\left[\left(x_{n}\right)_{n \in \mathbb{N}}\right] \in \widetilde{C_{0}(X)}$, because for each $\varepsilon \in(0,1)$ there is $n_{0} \in \mathbb{N}$ such that $M\left(x_{n}, x_{m}, \varepsilon / k\right)>1-\varepsilon / k$ for all $n, m \geq n_{0}$, so $H_{M}\left(T x_{n}, T x_{m}, \varepsilon\right)>1-\varepsilon$ for all $n, m \geq n_{0}$, which implies that $\left(T x_{n}\right)_{n \in \mathbb{N}}$ is a Cauchy sequence in $\left(C_{0}(X), H_{M}, *\right)$, and thus $\left[\left(T x_{n}\right)_{n \in \mathbb{N}}\right] \in \widetilde{C_{0}(X)}$.

Therefore, we have defined a multivalued mapping $\left.\widetilde{T}:(\widetilde{X}, \widetilde{M}, *) \rightarrow\left(\widetilde{C_{0}(X)}\right), \widetilde{H_{M}}, *\right)$ by

$$
\widetilde{T}\left[\left(x_{n}\right)_{n \in \mathbb{N}}\right]=\left[\left(T x_{n}\right)_{n \in \mathbb{N}}\right],
$$

for all Cauchy sequence $\left(x_{n}\right)_{n \in \mathbb{N}}$ in $(X, M, *)$. Note that, by Theorem 2.5 , we can consider $\widetilde{T}$ as a multivalued mapping from $\widetilde{X}$ to $C_{0}(\widetilde{X})$.

Then, we show the following.

2.9 Proposition. If $T$ is an $(\alpha, C)$-contraction on a fuzzy metric space $(X, M, *)$, then $\widetilde{T}$ is an $(\alpha, C)$-contraction on $(\widetilde{X}, \widetilde{M}, *)$.

Proof. Suppose that $\widetilde{M}(\widetilde{x}, \widetilde{y}, t)>1-t$, where $\widetilde{x}, \widetilde{y} \in \widetilde{X}$ and $t>0$. By left continuity of $\widetilde{M}(\widetilde{x}, \widetilde{y}, \cdot)$ at $t$, there exists $s_{t} \in(0, t)$ such that $\widetilde{M}\left(\widetilde{x}, \widetilde{y}, s_{t}\right)>1-s_{t}$. Put $\widetilde{x}:=$ $\left[\left(x_{n}\right)_{n \in \mathbb{N}}\right]$, and $\widetilde{y}:=\left[\left(y_{n}\right)_{n \in \mathbb{N}}\right]$. Then

$$
\sup _{0<s<s_{t}} \sup _{k \in \mathbb{N}} \inf _{n \geq k} M\left(x_{n}, y_{n}, s\right)>1-s_{t} .
$$

So, there exists $s_{0} \in\left(0, s_{t}\right)$ and $k_{0} \in \mathbb{N}$ such that $M\left(x_{n}, y_{n}, s_{0}\right)>1-s_{t}$ for all $n \geq k_{0}$. Hence

$$
M\left(x_{n}, y_{n}, s_{t}\right)>1-s_{t}
$$


for all $n \geq k_{0}$, and consequently

$$
H_{M}\left(T x_{n}, T y_{n}, \alpha s_{t}\right)>1-\alpha s_{t},
$$

for all $n \geq k_{0}$. Since $\alpha s_{t}<\alpha t$, we deduce that

$$
\sup _{0<s<\alpha t} \sup _{k \in \mathbb{N}} \inf _{n \geq k} H_{M}\left(T x_{n}, T y_{n}, s\right)>1-\alpha t
$$

so $\widetilde{H_{M}}(\widetilde{T} \widetilde{x}, \widetilde{T} \widetilde{y}, \alpha t)>1-\alpha t$. By Theorem $2.5, \widetilde{T}$ is an $(\alpha, C)$-contraction on $(\widetilde{X}, \widetilde{M}, *)$.

Combining propositions 2.7 and 2.9, we obtain the following.

2.10 Proposition. Let $(X, M, *)$ be a fuzzy metric space with $*_{L} \leq *$. If $T$ is an $(\alpha, C)$-contraction on $(X, M, *)$, then $\widetilde{T}$ has a fixed point.

2.11 Remark. In [23] Radu proved that every $C$-contraction on a complete fuzzy metric space has a unique fixed point. From this result and Proposition 2.9 it follows that if $f$ is a $C$-contraction on a fuzzy metric space $(X, M, *)$, then $\widetilde{f}$ has a unique fixed point in $\widetilde{X}$.

2.12 Remark. Observe that under the conditions of Proposition 2.10, the proof of Proposition 2.7 shows that for any $x_{0} \in X$ there are a sequence $\left(x_{n}\right)_{n \in \omega}$, with $x_{n+1} \in T x_{n}$ for all $n \in \omega$, and a $\widetilde{z} \in \widetilde{X}$ such that $\left(x_{n}\right)_{n \in \omega}$ converges to $\widetilde{z}$ with respect to $\tau_{\widetilde{M}}$, and $\widetilde{z} \in \widetilde{T z} \in C_{0}(\widetilde{X})$. Since, by Proposition $2.9, \widetilde{T}$ is an $(\alpha, C)$-contraction, we deduce that the sequence $\left(T x_{n}\right)_{n \in \omega}$ converges to $\widetilde{T z}$ with respect to $\tau_{H_{\widetilde{M}}}$, so with respect to $\tau_{\widetilde{H_{M}}}$, , by Theorem 2.5 . Note that we also have

$$
\lim _{n \rightarrow \infty} H_{\widetilde{M}}^{-}\left(\{\widetilde{z}\}, T x_{n}, t\right)=1 \quad \text { whenever } t>0 .
$$

\section{Application to metric spaces}

In this sort section we shall obtain the analogous metric of Theorem 2.5, with the help of the standard fuzzy metric. To this end, we will need the following two lemmas that may be found in [14, Proposition 1], and in [24, Example 5], respectively.

3.1 Lemma. Let $(X, d)$ be a metric space. Then $\widetilde{M}_{d}=M_{\widetilde{d}}$ on $\widetilde{X}$.

3.2 Lemma. Let $(X, d)$ be a metric space. Then $H_{M_{d}}=M_{H_{d}}$ on $C_{0}(X)$. 
In other words, we have the following commutative diagram:

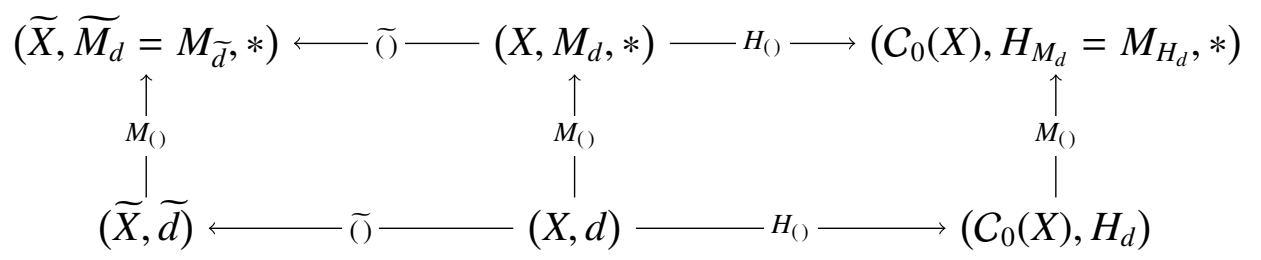

3.3 Theorem. Let $(X, d)$ be a metric space. Then:

(1) $\left(C_{0}(\widetilde{X}), H_{\widetilde{d}}\right)$ and $\left(\widetilde{C_{0}(X)}, \widetilde{H_{d}}\right)$ are isometric.

(2) $\left(\mathcal{K}_{0}(\widetilde{X}), H_{\widetilde{d}}\right)$ and $\left(\widetilde{\mathcal{K}_{0}(X)}, \widetilde{H_{d}}\right)$ are isometric.

Proof. By lemmas 3.1 and 3.2 we have $H_{\widetilde{M_{d}}}=H_{M_{\widetilde{d}}}=M_{H_{\widetilde{d}}}$ on $C_{0}(\widetilde{X})$ and $\widetilde{H_{M_{d}}}=$ $\widetilde{M_{H_{d}}}=M_{\widetilde{H_{d}}}$ on $\widetilde{C_{0}(X)}$.

By Theorem 2.5, there exists an isometry $\phi$ from $\left(C_{0}(\widetilde{X}), M_{H_{\widetilde{d}}}, *\right)$ onto $\left(\widetilde{C_{0}(X)}, M_{\widetilde{H_{d}}}, *\right)$. Hence $\phi$ is obviously an isometry from $\left(C_{0}(\widetilde{X}), H_{\widetilde{d}}\right)$ onto $\left(\widetilde{C_{0}(X)}, \widetilde{H_{d}}\right)$, which shows the assertion (1). The proof of assertion (2) follows similarly by Remark 2.6.

We can summarize the proof of the first item in the previous result in the following diagram:

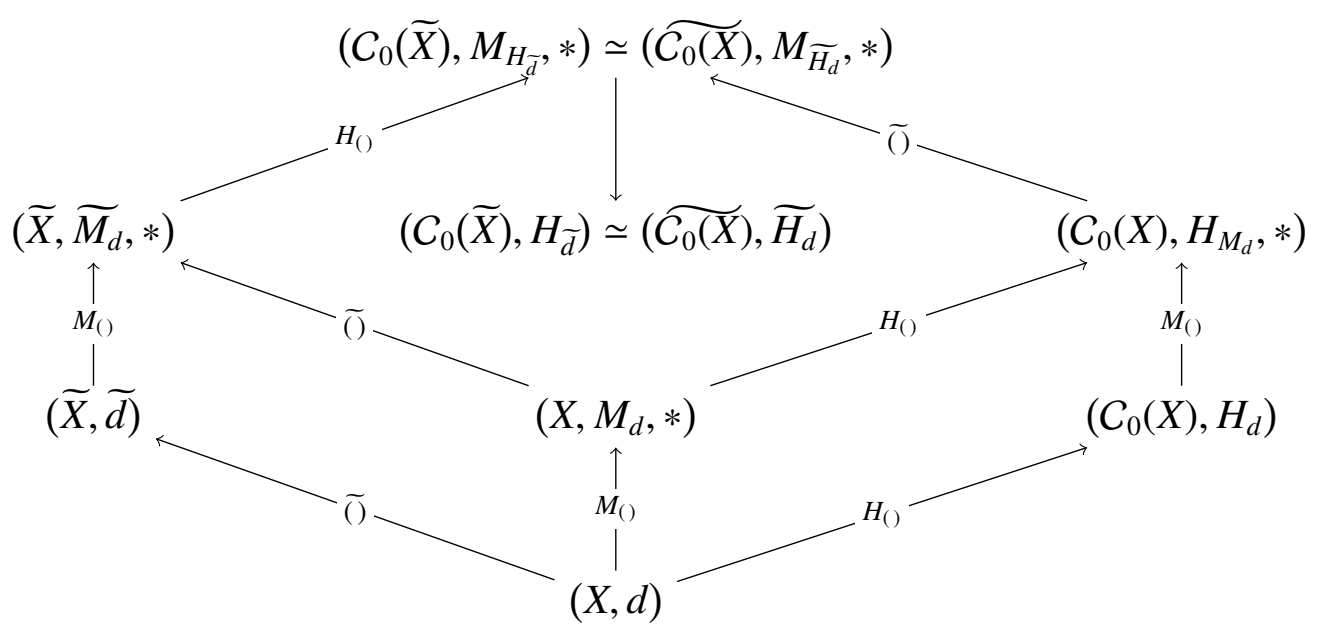




\section{Examples}

In this section we shall present some examples that illustrate our constructions and results given above.

4.1 Example. Let $X=(0,2)$ and let $(M, *=$ Prod) be the fuzzy metric on $X$ defined by $M(x, y, 0)=0$ and

$$
M(x, y, t)=\frac{\min \{x, y\}}{\max \{x, y\}}
$$

for all $t>0$. Observe that $(X, M, *)$ is not complete because the sequence (2 $\left.2^{-n}\right)_{n \in \mathbb{N}}$ is Cauchy but does not converge in $(X, M, *)$. Hence $\left(C_{0}(X), H_{M}, *\right)$ is not complete.

Now put $A_{n}=\left[1+\frac{1}{n}, 2-\frac{1}{n}\right]$ for all $n \in \mathbb{N}$ with $n \geq 3$. Clearly $A_{n} \in C_{0}(X)$. Furthermore $\left(A_{n}\right)_{n \geq 3}$ is a Cauchy sequence in $\left(C_{0}(X), H_{M}, *\right)$. Indeed, for all $n, m \in$ $\mathbb{N}(n \geq 3)$ and $t>0$ we have $H_{M}^{-}\left(A_{n}, A_{n+m}, t\right)=1$ because $A_{n} \subsetneq A_{n+m}$. Moreover, if $x \in A_{n+m} \backslash A_{n}$ and $x \in\left[1+\frac{1}{n+m}, 1+\frac{1}{n}\right)$, an easy computation shows that $M\left(A_{n}, x, t\right)=$ $x /\left(1+\frac{1}{n}\right)$. On the contrary, for $x \in A_{n+m} \backslash A_{n}$ with $x \in\left(2-\frac{1}{n}, 2-\frac{1}{n+m}\right]$, we obtain $M\left(A_{n}, x, t\right)=\left(2-\frac{1}{n}\right) / x$, so

$$
H_{M}^{+}\left(A_{n}, A_{n+m}, t\right)=\inf _{x \in A_{n+m}} M\left(A_{n}, x, t\right)=\min \left\{\frac{1+\frac{1}{n+m}}{1+\frac{1}{n}}, \frac{2-\frac{1}{n}}{2-\frac{1}{n+m}}\right\}=\frac{n(n+m+1)}{(n+1)(n+m)} .
$$

It immediately follows that $\left(A_{n}\right)_{n \geq 3}$ is a Cauchy sequence in $\left(C_{0}(X), H_{M}, *\right)$. Therefore there exists $\widetilde{A} \in \widetilde{C_{0}(X)}$ such that $\left(A_{n}\right)_{n \geq 3}$ converges to $\widetilde{A}$ in $\left(\widetilde{C_{0}(X)}, \widetilde{H_{M}}, *\right)$. By Theorem 2.5, we can compute the distance $\widetilde{H_{M}}\left(\widetilde{A}, A_{n}, t\right)$ in an easier fashion by calculating $H_{\widetilde{M}}\left(\widetilde{A}, A_{n}, t\right)$. To this end, we first note that the fuzzy metric space $\left((0,2], M_{e}, *\right)$ is complete (compare e.g. [8, Example 11.4.1]), where $M_{e}$ is the extension to $(0,2]$ of $M$. Clearly $\left((0,2], M_{e}, *\right)$ is (isometric to) the completion of $(X, M, *)$. Hence, we can suppose that $[1,2] \in \mathcal{C}_{0}(\widetilde{X})$. Furthermore, the sequence $\left(A_{n}\right)_{n \geq 3}$ converges to $[1,2]$ in $\left(C_{0}(\widetilde{X}), \widetilde{M}, *\right)$, because for each $n \geq 3$ and $t>0$, we have

$$
H_{M_{e}}^{-}\left([1,2], A_{n}, t\right)=\min \left\{\frac{1}{1+\frac{1}{n}}, \frac{2-\frac{1}{n}}{2}\right\}=\frac{n}{n+1} \quad \text { and } \quad H_{M_{e}}^{+}\left([1,2], A_{n}, t\right)=1 .
$$

Consequently

$$
\widetilde{H_{M}}\left(\widetilde{A}, A_{n}, t\right)=H_{M_{e}}\left([1,2], A_{n}, t\right)=\frac{n}{n+1},
$$

for each $n \geq 3$ and each $t>0$. 
In our next example we shall use the following well-known construction.

If $(X, d)$ is a (complete) metric space, then the triple $\left(X, M_{d, 01}, \wedge\right)$ is a (complete) fuzzy metric space, where $M_{d, 01}$ is defined by $M_{d, 01}(x, y, t)=1$ if $d(x, y)<t$ and $M_{d, 01}(x, y, t)=0$ if $d(x, y) \geq t$. Moreover the topologies induced by $d$ and $\left(M_{d, 01}, \wedge\right)$ coincide. We shall refer to $\left(X, M_{d, 01}, \wedge\right)$ as the 01 -fuzzy metric space induced by $(X, d)$.

4.2 Example. Let $\Sigma$ be an alphabet, i.e., a non-empty set. As usual, the elements of $\Sigma$ are called letters, or symbols, and we denote by $\Sigma^{F}$ the set of all finite sequences of letters over $\Sigma$, and by $\Sigma^{\omega}$ the set of all infinite sequences of letters over $\Sigma$. We assume that the empty sequence, or sequence of zero letters, $\varepsilon$ is an element of $\Sigma^{F}$.

Let $\Sigma^{\infty}=\Sigma^{F} \cup \Sigma^{\omega}$, and denote by $\sqsubseteq$ the prefix order on $\Sigma^{\infty}$, i.e., $x \sqsubseteq y$ if and only if $x$ is a prefix of $y$. Thus $\varepsilon \sqsubseteq y$ for every $y \in \Sigma^{\infty}$.

Let $\ell(x)$ denotes the length of $x \in \Sigma^{\infty}$. Then $\ell(x) \in[1,+\infty]$ whenever $x \neq \varepsilon$, and $\ell(\varepsilon)=0$. For all $x, y \in \Sigma^{\infty}$ let $x \sqcap y$ be the common prefix of $x$ and $y$.

We also recall that the Baire metric, or the Baire distance, on $\Sigma^{\infty}$ is the metric $d_{B}$ defined, for each $x, y \in \Sigma^{\infty}$, by $d_{B}(x, y)=0$ if $x=y$, and $d_{B}(x, y)=2^{-\ell(x \sqcap y)}$ otherwise.

It is well known (see e.g. [3, Theorem 1.29]) that $\left(\Sigma^{\infty}, d_{B}\right)$ is a complete metric space. In fact, it is (isometric to) the completion of the metric space $\left(\Sigma^{F}, d_{B}\right)$.

Now consider the 01-fuzzy metric spaces $\left(\Sigma^{F}, M_{d_{B}, 01}, \wedge\right)$ and $\left(\Sigma^{\infty}, M_{d_{B}, 01}, \wedge\right)$, induced by $\left(\Sigma^{F}, d_{B}\right)$ and $\left(\Sigma^{\infty}, d_{B}\right)$, respectively. Then $\left(\Sigma^{\infty}, M_{d_{B}, 01}, \wedge\right)$ is (isometric to) the completion of $\left(\Sigma^{F}, M_{d_{B}, 01}, \wedge\right)$ (see e.g., [7, Example 4]). So $\left(C_{0}\left(\Sigma^{\infty}\right), H_{M_{d_{B}}, 01}, \wedge\right)$ can be identified with $\left(C_{0}\left(\widetilde{\Sigma^{F}}\right), H_{\widetilde{d_{B}}, 01}, \wedge\right)$. Note also that $\left(M_{d_{B}, 01}, \wedge\right)$ induces the discrete topology on $\Sigma^{F}$.

Let $\mathcal{F}$ be the class of all self mappings $f$ of $\Sigma^{F}$ satisfying the following two conditions for each $x, y \in \Sigma^{F}$ :

(1) $\ell(f(x))=\ell(x)+1$,

(2) $\ell(f(x \sqcap y)) \leq \ell(f(x) \sqcap f(y))$.

We point out that this kind of self mappings plays a crucial role in applying fixed point methods to discuss the existence and uniqueness of solution for recurrence equations that are typically associated to certain algorithms (see e.g. [6, 25, 26, 27]).

In fact, this kind of questions suggests the following construction.

Given $f \in \mathcal{F}$ define the multivalued mapping $T: \Sigma^{F} \rightarrow \mathcal{C}_{0}\left(\Sigma^{F}\right)$ given by

$$
T x=\left\{f^{n}(x): n \in \mathbb{N}\right\},
$$


for all $x \in \Sigma^{F}$.

We shall prove that there is $z \in \Sigma^{\omega}$ with $\{z\}=\widetilde{T} z$, and such that for any $x_{0} \in \Sigma^{F}$, the sequence $\left(f^{n}\left(x_{0}\right)\right)_{n \in \omega}$ converges to $z$.

To this end we first show that $T$ is an $(1 / 2, C)$-contraction on $\left(\Sigma^{F}, M_{d_{B}, 01}, \wedge\right)$.

Suppose that $M_{d_{B}, 01}(x, y, t)>1-t$, for $x, y \in \Sigma^{F}$ with $x \neq y$, and $t>0$.

Then $M_{d_{B}, 01}(x, y, t)=1$, because if $M_{d_{B}, 01}(x, y, t)=0$, we deduce that $t>1$ and $d_{B}(x, y) \geq t$, which contradicts that $d_{B}(x, y) \leq 1$. Hence $d_{B}(x, y)<t$, i.e., $2^{-\ell(x \sqcap y)}<t$.

Now we show that $H_{M_{d_{B}, 01}}(T x, T y, t / 2)=1$. Indeed, for each $n \in \mathbb{N}$ we have, by condition (1), that $\ell\left(f^{n}(x \sqcap y)\right)=\ell(x \sqcap y)+n$, so, by condition (2),

$$
\ell(x \sqcap y)+n \leq \ell\left(f^{n}(x) \sqcap f^{n}(y)\right) .
$$

Hence

$$
d_{B}\left(f^{n}(x), f^{n}(y)\right) \leq 2^{-(\ell(x \sqcap y)+n)} \leq 2^{-(\ell(x \sqcap y)+1)}<t / 2 .
$$

Fix $s_{0} \in\left(2^{-(\ell(x \sqcap y)+1)}, t / 2\right)$. Then, for each $n \in \mathbb{N}$,

$$
M_{d_{B}, 01}\left(f^{n}(x), f^{n}(y), s_{0}\right)=1,
$$

so

$$
M_{d_{B}, 01}\left(f^{n}(x), T y, s_{0}\right)=1
$$

and consequently

$$
H_{M_{d_{B}, 01}}^{-}(T x, T y, t / 2)=\sup _{0<s<t / 2} \inf _{n \in \mathbb{N}} M_{d_{B}, 01}\left(f^{n}(x), T y, s\right)=1 .
$$

Similarly we obtain that $H_{M_{d_{B}, 01}}^{+}(T x, T y, t / 2)=1$.

We conclude that $T$ is an $(1 / 2, C)$-contraction on $\left(\Sigma^{F}, M_{d_{B}, 01}, \wedge\right)$.

Since, in particular, $M_{d_{B}, 01}\left(f(x), f(y), s_{0}\right)=1$, we deduce that $f$ is a $C$-contraction for $\alpha=1 / 2$.

Notice that, by Remark 2.11, $\widetilde{f}$ has a unique fixed point $z \in \Sigma^{\infty}$. Then, Proposition 2.10 and Remark 2.12 tell us that for each $x_{0} \in \Sigma^{F}$ the sequence $\left(f^{n}\left(x_{0}\right)\right)_{n \in \omega}$, converges to $z$. (Observe that actually $z \in \Sigma^{\omega}$ because $\ell\left(f^{n}\left(x_{0}\right)\right) \geq n$ for all $n \in \mathbb{N}$ ).

It remains to prove that $\{z\}=\widetilde{T} z$. To this end, choose $x_{0} \in \Sigma^{F}$ and put $x_{n}=f^{n}\left(x_{0}\right)$ for all $n \in \omega$. Since $\left(x_{n}\right)_{n \in \omega}$ converges to $z$ in $\left(\Sigma^{\infty}, M_{d_{B}, 01}, \wedge\right)$ and $\widetilde{T}$ is a $(1 / 2, C)$-contraction, it follows that the sequence $\left(T x_{n}\right)_{n \in \omega}$ converges to $\widetilde{T} z$ in $\left(C_{0}\left(\Sigma^{\infty}\right), H_{M_{d_{B}}, 01}, \wedge\right)$ (see Remark 2.12). Next we show that $\left(T x_{n}\right)_{n \in \omega}$ also converges to $\{z\}$ in $\left(C_{0}\left(\Sigma^{\infty}\right), H_{M_{d_{B}}, 01}, \wedge\right)$. For each $t>0$ there exists $n_{t} \in \mathbb{N}$ such that 
$M_{d_{B}, 01}\left(z, x_{n}, t\right)=1$ for all $n \geq n_{t}$, thus $2^{-\ell\left(z \sqcap x_{n}\right)}<t$ for all $n \geq n_{t}$. Hence, if $y \in T x_{n}$, $n \geq n_{t}$, then for $s \in\left(2^{-\ell\left(z \sqcap x_{n_{t}}\right)}, t\right)$ we have $M(z, y, s)=1$. Indeed, by definition of $T, y=f^{j}\left(x_{n}\right)$ for some $j \in \mathbb{N}$, and consequently $y=x_{n+j}$. Therefore

$$
H_{M_{d_{B}, 01}}^{-}\left(\{z\}, T x_{n}, t\right)=\sup _{0<s<t} M\left(z, T x_{n}, s\right)=1,
$$

and

$$
H_{M_{d_{B}, 01}}^{+}\left(\{z\}, T x_{n}, t\right)=\sup _{0<s<t} \inf _{y \in T x_{n}} M(\{z\}, y, s)=1,
$$

for all $n \geq n_{t}$. Hence $\left(T x_{n}\right)_{n \in \omega}$ converges to $\{z\}$ in $\left(C_{0}\left(\Sigma^{\infty}\right), H_{M_{d_{B}}, 01}, \wedge\right)$.

On th other hand, we know, by Remark 2.12 , that $\left(T x_{n}\right)_{n \in \omega}$ converges to $\widetilde{T} z$ in $\left(C_{0}\left(\Sigma^{\infty}\right), H_{M_{d_{B}}, 01}, \wedge\right)$. Consequently $\{z\}=\widetilde{T} z$.

By a dynamical system it is understood a pair $(X, f)$, with $X: \equiv(X, d)$ a metric space, and $f$ a continuous function from $X$ into itself. Given a dynamical system $(X, f)$, two points $x, y \in X$ are called proximal if for every $\varepsilon>0$, infinitely many $n \in \mathbb{N}$ satisfy $d\left(f^{n}(x), f^{n}(y)\right)<\varepsilon$ where, as usual, $f^{n}$ stands for the $n$-iterate of $f$.

In the realm of dynamical systems, the Stone-Čech compactification $\beta(\mathbb{N})$ of the discrete space $\mathbb{N}$ of the natural numbers plays an important role by means of the notion of $p$-limit point. If $\beta(\mathbb{N})$ is viewed as the space of all ultrafilters on $\mathbb{N}$, then, for each free ultrafilter $p \in \beta(\mathbb{N}) \backslash \mathbb{N}$, a point $x \in X$ is called a $p$-limit point of an orbit $\left(f^{n}(y)\right)_{n \in \mathbb{N}}$ if, for every neighborhood $V$ of $x,\left\{n \in \mathbb{N}: f^{n}(y) \in V\right\} \in p$.

For some applications of the notion of $p$-limit point in dynamical system theory see, for instance, $[1,5,10]$. Recall that we can associate to each dynamical system $(X, f)$ another dynamical system $\left(\mathcal{K}_{0}(X), 2^{f}\right)$, with $\mathcal{K}_{0}(X): \equiv\left(\mathcal{K}_{0}(X), H_{d}\right)$, where $2^{f}$ is the continuous extension of $f$ to $\mathcal{K}_{0}(X)$ defined as

$$
2^{f}(A)=\bigcup\{f(x): x \in A\} .
$$

Observe that $\left(2^{f}\right)^{n}$ coincides with $2^{f^{n}}$.

4.3 Example. Let $(X, f)$ be a dynamical system and assume that $f$ is a contractive function. It is a well-known fact that $2^{f}: \mathcal{K}_{0}(X) \rightarrow \mathcal{K}_{0}(X)$ is contractive as well. Notice that contractiveness implies that $H_{d}\left(2^{f^{n}}(A), 2^{f^{n}}(B)\right)$ converges to zero so that any two compact subsets $A$ and $B$ in $\mathcal{K}_{0}(X)$ are proximal in a strong way. However, the previous theorem allows us to describe the situation by means of $p$-limits points. To see this, observe that Theorem 3.3 permits us to consider $\left(2^{f}, \mathcal{K}_{0}(X)\right)$ as a subsystem of $\left(2^{\widetilde{f}}, \mathcal{K}_{0}(\widetilde{X})\right)$. Since $2^{\widetilde{f}}$ is also contractive, Banach 
fixed point theorem tells us that the sequence $\left(2^{f^{n}}(A)\right)_{n \in \mathbb{N}}$ converges to the unique fixed point, say $K$, of $2^{\widetilde{f}}$. Thus, for all $p \in \beta(\mathbb{N}) \backslash \mathbb{N}, K$ is the unique $p$-limit point of the orbit $\left(2^{f^{n}}(A)\right)_{n \in \mathbb{N}}$ for every $A \in \mathcal{K}_{0}(X)$ (in fact, $2^{f^{n}}(A)=2^{f^{n}}(A)$ for all $n \in \mathbb{N}$ ).

\section{Conclusions}

We present a description of the completion of the Hausdorff fuzzy metric space on the collection of all non-empty closed subsets of a fuzzy metric space in the sense of Kramosil and Michalek, which allows us its application in a natural and easy way: we give some examples pointing out this fact. Considering the usefulness of the theory of hyperspaces in several branches of Mathematics and Computer Sciences, our approach provides a way for future researches in this field. It is worth noting that our outcome permits us to obtain the analogous result for metric spaces.

Acknowledgement. The authors are grateful to the reviewers for several useful suggestions and comments.

\section{References}

[1] E. Akin, Recurrence in Topological Dynamics. Furstenberg Families and Ellis Actions. The University Series in Mathematics, Plenum Press, New York, 1997.

[2] G. Artico, R. Moresco, Notes on the topologies and uniformities of hyperspaces, Rend. Sem. Mat. Univ. Padova 63 (1980), 51-60.

[3] J. de Bakker, E. de Vink, Control Flow Semantics. Foundations of Computing Series, The MIT Press, Cambridge, 1996.

[4] G. Beer, Topologies on Closed and Closed Convex Sets. Kluwer Academic Publishers, Dordrecht, 1993.

[5] A. Blass, Ultrafilters: where topological dynamics = algebra = combinatorics. Topology Proc. 18 (1993), 33-56.

[6] F. Castro-Company, S. Romaguera, P. Tirado, The Banach Contraction Principle on Fuzzy Quasi-Metric Spaces and on Product Complexity Spaces: Two Approaches to Study the Cost of Algortihms with a Finite System of 
Recurrence Equations, Stud. Comput. Intell., Lecture Notes in Computer Science, vol. 399, Springer-Verlag, 2012, pp. 253-268.

[7] F. Castro-Company, S. Romaguera, P. Tirado, The bicompletion of fuzzy quasi-metric spaces, Fuzzy Sets Syst. 166 (2011), 56-64.

[8] Y.J. Cho, M. Grabiec, V. Radu, On Nonsymmetric Topological and Probabilistic Structures. Nova Science Publishers, New York, 2006.

[9] R.J. Egbert, Products and quotients of probabilistic metric spaces, Pacific J. Math. 24 (1968), 437-455.

[10] S. García-Ferreira, M. Sanchis, Ultrafilter-limit points in metric dynamical systems. Comment. Math. Univ. Carolin. 48 (2007), no. 3, 465-485.

[11] A. George, P. Veeramani, On some results in fuzzy metric spaces, Fuzzy Sets Syst. 64 (1994), 395-399.

[12] A. George, P. Veeramani, On some results of analysis of fuzzy metric spaces, Fuzzy Sets Syst. 90 (1997), 365-368.

[13] V. Gregori, S. Romaguera, Some properties of fuzzy metric spaces, Fuzzy Sets Syst. 115 (2000), 485-489.

[14] V. Gregori, S. Romaguera, On completion of fuzzy metric spaces, Fuzzy Sets Syst. 130 (2002), 399-404.

[15] O. Hadžić, E. Pap, A fixed point theorem for multivalued mappings in probabilistic metric spaces and an application in fuzzy metric spaces, Fuzzy Sets Syst. 127 (2002), 333-344.

[16] T.L. Hicks, Fixed point theory in probabilistic metric spaces, Univ. u Novom Sadu Zb. Rad. Prirod.-Mat. Fak. Ser. Mat. 13 (1983), 63-72.

[17] N.R. Howes, Modern Analysis and Topology. Springer-Verlag, New York, 1995.

[18] E.P. Klement, R. Mesiar, E. Pap, Triangular Norms, Kluwer Academic Publishers, Dordrecht, 2000.

[19] I. Kramosil, J. Michalek, Fuzzy metrics and statistical metric spaces, Kybernetika 11 (1975), 326-334. 
[20] E. Michael, Topologies on spaces of subsets, Trans. Amer. Math. Soc. 71 (1951), 152-182.

[21] S.B. Nadler Jr., Multi-valued contraction mappings. Pacific J. Math. 30 (1969), 475-488.

[22] E. Pap, O. Hadžić, R. Mesiar, A fixed point theorem in probabilistic metric spaces and an application, J. Math. Anal. Appl. 202 (1996), 433-449.

[23] V. Radu, Some fixed point theorems in $P M$-Spaces, Lecture Notes Math. 1233 (1987), 125-133.

[24] J. Rodríguez-López, S. Romaguera, J.M. Sánchez-Álvarez, The Hausdorff fuzzy quasi-metric, Fuzzy Sets Syst. 161 (2010), 1078-1096.

[25] S. Romaguera, A. Sapena, P. Tirado, The Banach fixed point theorem in fuzzy quasi-metric spaces with application to the domain of words, Topology Appl. 154 (2007), 2196-2203.

[26] S. Romaguera, P. Tirado, Contraction maps on ifqm-spaces with application to recurrence equations of Quicksort, Elect. Notes Theoret. Comput. Sci. 225 (2009), 269-279.

[27] R. Saadati, S.M. Vaezpour, Y.J. Cho, Quicksort algorithm: Application of a fixed point theorem in intuitionistic fuzzy quasi-metric spaces at a domain of words, J. Comput. Appl. Math. 228 (2009), 219-225.

[28] B. Schweizer, A. Sklar, Statistical metric spaces, Pacific J. Math. 10 (1960), 314-334.

[29] C. Sempi, Hausdorff distance and the completion of probabilistic metric spaces, Boll. U.M.I. 6-B (1992), 317-327.

[30] H. Sherwood, On the completion of probabilistic metric spaces, Z. Wahrsch. verw. Geb. 6 (1966), 62-64.

[31] R.M. Tardiff, Topologies for probabilistic metric spaces, Pacific J. Math. 65 (1976), 233-251. 